\title{
Models of attention
}

\section{Moyra Derby, University for the Creative Arts}

\begin{abstract}
In this article the question posed by Yve-Alain Bois via Hubert Damisch in Painting as Model, 'what is the mode of thought of which painting is the stake?' (1990) is shifted to what is the mode of attention? Informed by current cognitive and neuropsychological research, paintings combative art history is assessed through the lens of attention. The unravelling of modernist painting is proposed as a conflict between models of attention, and divergent attentional expectations. Modernist values of immediacy, presentness, wholeness are considered conditions of an ideal attentional experience, one that attempts to hold back a partial, fragmented and distracted counter experience of modernity. Painting as Model argued for painting's specificity, pulling away from a formalism that reduces painting to the visual, and theoretical structures that bypass the made object. This article proposes an attentional-specificity for painting; the limits of attentional capacity, distinctions between focused, distributed and divided attention correlating with the cognitive complexity held by the structural, spatial and material conditions of painting.
\end{abstract}

\section{Keywords}

aesthetic experience

distributed attention

focused attention

Frank Stella

painting

Painting as Model

Yve-Alain Bois 
In Yve-Alain Bois's text Painting as Model, the opening question is from Hubert Damisch, 'What does it mean for a painter to think?' and then 'Not only what is the role of speculative thought for the painter at work? but above all what is the mode of thought of which painting is the stake?' (Bois 1990: 245).

I want to adopt and adapt that question, in order to ask, what is the mode of attention of which painting is the stake? This slide from mode of thought to mode of attention is not meant as a verbal sleight of hand, but it is a move sideways, in order to formulate a response to an impossibly divisive choice that Painting as Model is also committed to discounting. Between the visual or the conceptual, between formal or theoretical priorities for painting, and an acknowledgement that in such a standoff the material properties of a thing made to be looked at get edged out. The art historical backdrop of Painting as Model is the unravelling of modernist painting, as though confounded by its own logics, and I'm interested in the part that divergent and unsustainable attentional expectations play in that unravelling.

The significance of Bois's writing in Painting as Model, for a painter, is the regard for the materiality of painting and detailed observation of method, and of taking painting seriously on its own terms. My proposition is that part of painting's specificity is its investment in capturing and conditioning attention, the capture and thread of attention caught in the press or drag of a worked surface. That attention refuses a division between optical and cognitive processing.

This article will be accompanied by an image of Frank Stella in his studio, photographed by Hollis Frampton presumably in 1959 when the painting in process was finished. I will reproduce the 
image twice, and it will keep me company, though I will not refer to Stella's work or working method until the very end. It acts for now as a marker in time ${ }^{1}$.

${ }^{1}$ Hollis Frampton's documentation of Frank Stella in his studio in the late 1950's fulfils several functions here. As an image of work in progress it maintains the focus on the material object of painting, and the attentiveness of method. It pictures attention, and repeated it also activates the attentional process of comparison for the reader. Additionally, it takes up a slot in an art historical timeline; the confidence and tight logics of American formalism seem emphatically realized in Stella's Black Paintings, so the documentation can stand for a position that Bois seeks to counter and clarify in Painting as Model, making a clear distinction between European and American formalism in order to reclaim some ground. It can also trigger an associative link to Stella's later work, work that was contemporary to the publication of Painting as Model in 1989 or 1990, its hyper pictorial complexity acting as self generated evidence of the unsustainability of the tight logics spun in the late 1950's and early 1960's. However, despite my sense of the relevance of this reference, Frank Stella is only mentioned three times in the Painting as Model collection of essays; once as an aside, once in brackets, and once in a footnote (Bois 1990: 123, 181, 310n15). As a visual accompaniment to this article, Frampton's photograph of Stella at work also registers on the periphery; I do not refer to Stella's work in this text until the end, and then only briefly. This peripheral status is intentional, indicating the process of attentional capture and distraction that painting participates in, and a pull within a work to something outside of it. 


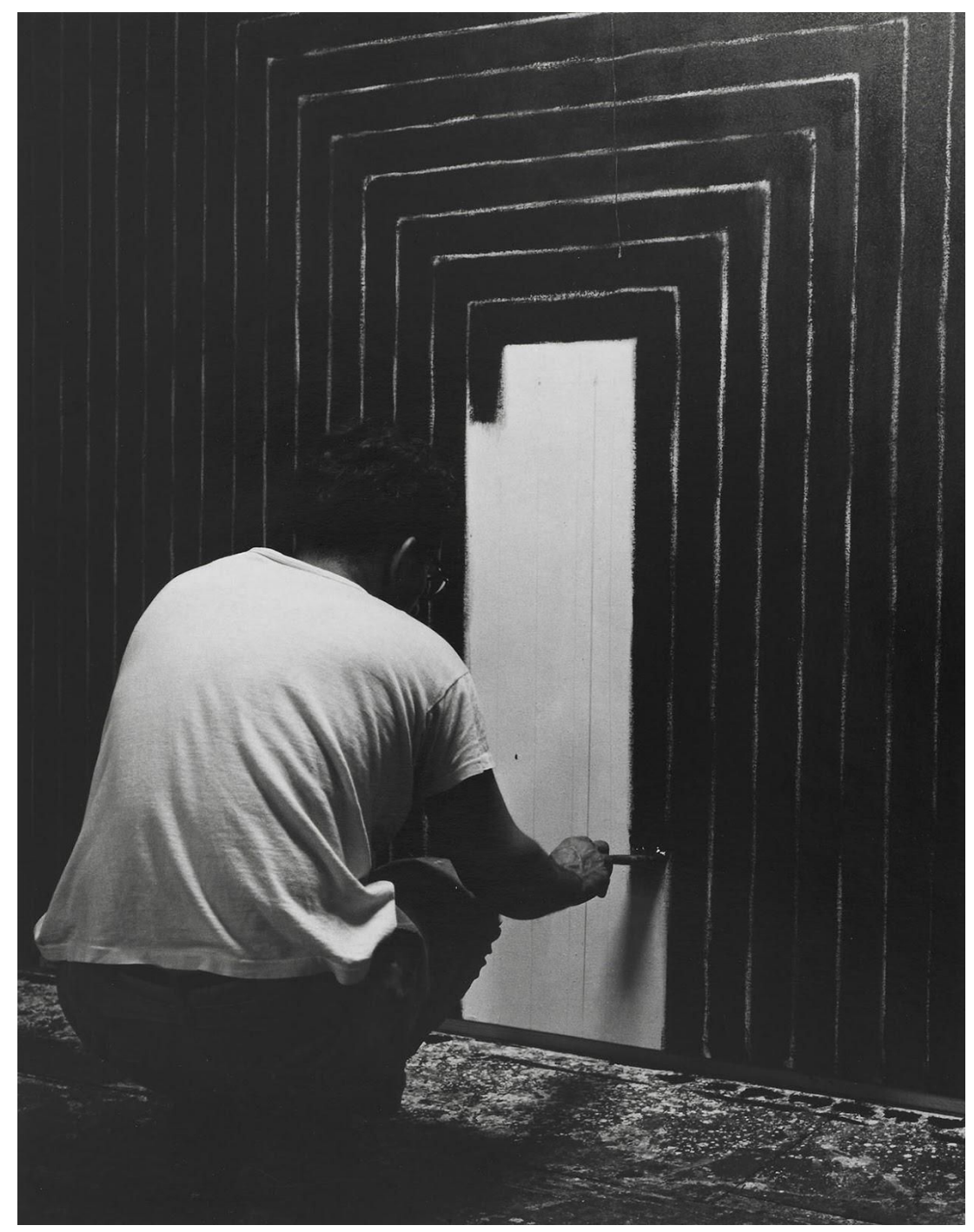

Figure 1: Hollis Frampton, untitled from The Secret World of Frank Stella, 1958-62,

black-and-white photograph, $10 \times 8$ ", Walker Art Center, Minneapolis. (C) Estate of Hollis Frampton.

Attention is widely understood as the selective function of perception, and I will add speculative to that description, retaining Damisch's notion of speculative thought. Attention modulates perception. As a response mechanism to external stimuli, attention is culturally and contextually dependent. Current research makes it clear that attention is variable, pliable, capacity dependent, and episodic. Attention frames and limits in much the way a picture does. We might even call attention reductive, in the sense that reductiveness is a pragmatic strategy for working with an 
attentional capacity which can be exceeded. We could say that pictorial conventions reflect our experience of attention, or that a dominance of pictorial modes impacts our visualization of attention. M. L. Marillier at the end of the nineteenth century defined attention as 'a state of consciousness which is the result of the temporary predominance of one representation over the representations which are co-existent with it at any given moment' (Hamlin 1896). As recently defined by Jesse Prinz, attention is the process which makes a representation available to working memory, and the processes of attention and the processes of memory and imagination are closely connected, with attention considered a precondition for conscious thought (2012).

Within the fabricated surface offered by a painting, we have a reduced and structured version of a field of vision. Visual stimuli are pre-set although they may be optically unstable, materially ambiguous or spatially unresolvable. And the selective impulse of attention, to target relevance over distractors, is challenged in a particular way by the intentionality of painting. Salience-based shifts in attention provide the basis for composition and counter motivations of non-composition; colour, tonal contrast, edge, all making various plays for our attention. Some shifts in attention are reactive and involuntary, so that high contrast or high chroma stimuli unavoidably catch our eye. Other shifts are emotionally or motivationally dependent, as we cross compare for likeness, or correspondance (Franconeri et al. 2004) (Yantis and Jonides 1984). We know that attention is susceptible to suggestion, and directed attention can introduce a task induced bias so that it is possible to miss what is right before your eyes. The temporality of attention, and the miniscule but measurable time lag in processing means attention is also characterized by gaps and glitches.

Assessing attentional engagement has a subtle complexity, as the speculative processes of attention can be overt or covert. Overt attention is trackable with approximation by following the direction of a gaze, covert attention indicates when focus turns inwards, and the correlation 
between what we are looking at and what we are paying attention to gets lost. This introversion of attention is hard to catch hold of, as it moves through recognition and association into reverie, the encoding and recall of memory. A distinction made since the 1970's in neuropsychological research has been between focused and distributed attention, or alternatively referred to as focal and diffuse attention. This distinction has been shown to be crucial in our visual sense of number, specifically the difference between counting and estimating. The need to individuate in order to count, the focal deployment of one after the other, requires the exactness of focused attention. Whereas distributed attention enables swift approximation, a sense of the overall statistical properties of a given scene (Chong and Evans 2011). 'The deployment of attention determines what we see' (Treisman 2006) and in turn what we remember, and the flexibility between modes of attention enables us to grasp both the gist and the detail.

So we can say that the mechanisms of attention within a space demarcated as painting will be multiple, cognitively complex, subjective, temporal and speculative rather than fixed and determined. Attention is enmeshed in a value system. It has a social, economic and cultural function; attention is active in our compliance as citizens, attainment as learners, productivity as workers and receptiveness as consumers. Hyper attention or continuous partial attention are recent terms that seek to characterize our attentional response to multiple, and updating demands on our attentional resources in our negotiations with attention needy technologies (Hayles 2007; Stone 1998). Whether these responses are evidence of an adaptive attentional flexibility, or evidence of a deficit, or lack of depth or duration in our attentional capacity, seems dependent on context. For Katherine Hayles hyper attention, and its networked connectivity, is framed in contrast to deep attention and marks a generational divide in cognitive modes (Hayles 2007). William James's nineteenth century definition of attention already positioned it in relation to a fear over its loss, so the clarity, focalization and concentration he associates with attention is 
contrasted with what James called the 'confused, dazed and scatter-brained state of distraction' (1890). James goes on to describe the lethargy of an inattentive state, but twenty-first century accounts of a crisis in attention emphasize a hyper, partial, agitated sense of attention under duress. Attention's finite capacity, its' alertness to change and difference, the subjectivity of attention's selectivity, makes it seem especially vulnerable in the rush and hustle of what is now often termed an attention economy, when unfathomable quantities of information compete for the capacity and time constrained resources of our attention (Terranova 2012).

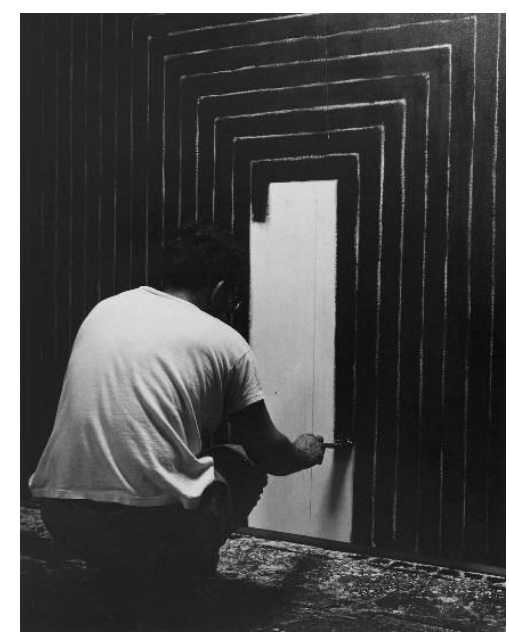

Hollis Frampton, untitled from The Secret World of Frank Stella, 1958-62, black-and-white photograph, $10-8$ ", Walker Art Center, Minneapolis. (C) Estate of Hollis Frampton.

I am proposing painting as a structure that is invested in capturing and conditioning attention, and given the variability of attentional processes, their precariousness and flexibility, their social and economic function, this maps a complex field. However, it is a more limited or ideal model of attention that painting is most often associated with. We can track the art historical lineage of an idealized model of attention through the terms contemplation, beholding, absorption, presentness, and they have their roots in western painting's function within structures of belief and power. 
In the nineteenth century and twentieth century this model of attention is condensed further by painting's concentration on formal properties, a reductiveness that can be seen in parallel to attention's task of synthesis. This synthesis or perceptual wholeness gets set like a counterbalance to combat a fragmented attentional experience, what Walter Benjamin described as 'reception in a state of distraction', a by-product of the technological, cultural and social shifts that start with industrialization and escalate in a digital multiplicity (Duttlinger 2007). Jonathan Cray's contention is "that modern distraction was not a disruption of stable or "natural" kinds of sustained, value-laden perception, [...] [...] but was an effect, and in many cases a constituent element, of the many attempts to produce attentiveness in human subjects' (Crary 1999: 49).

Crary's focus on nineteenth century technologies that produce a sort of synthesis on our behalf, and social and economic structures that required new modes of attentive engagement, have resonance for our contemporary concerns over the modification of our attention through the back lit interface of an endlessly updating stream of information. David Joselit also articulates a position for painting and its long held investment in negotiating attention within the onslaught of image production and image circulation, while also offering a resistance. Joselit uses the word speculation as 'a type of vision indigenous to circulation in that it aims to foresee, even to regulate, how an artwork will behave once it enters the world' (2016: 19). That painting might point outside of itself, defer capture, that the non-compliance of objectness and material drag could act like glitches in a smooth stream of an optically based consumption.

However, the criteria for a model of attention for painting has most often been honed in isolation from other attentional experiences. Aesthetic sensibility describes a form of idealized attentive engagement and was first formulated in detail in the eighteenth century with German philosopher Alexander Baumgarten's Aesthetica of 1750, and refers to our experiences of bodily perception 
and sensation but in separation from conceptual thought. This demarcation between the perceptual and the conceptual is underscored with Immanuel Kant, with the subjectivity of aesthetic judgement seen in separation from cognitive complexity, and ethical and political concerns. Out of what we can assume is a genuine attempt by eighteenth century thinkers to give proper regard to a sensory mode of judgement, we see the divide between a visual and a conceptual reading of painting being constructed before our eyes.

In Kant's formulations, disinterestedness and universality are key criteria. Disinterestedness in the sense of a pleasure that is impartial, non-personal and distanced, universality in the sense of unarguably true. Universality leads to an association between aesthetic experience and immediacy, because that which is universally true must of course be apparent at once, rather than inferred by concepts, or figured out in any complicated way. Additionally, according to Kant, for an object to qualify for aesthetic engagement, a balance needs to be achieved between a recognition of purpose or intent, and a condition of being without purpose, withdrawing art from the utility of objecthood, while also holding on to a purposefulness through the register of intentionality. Finally aesthetic judgement needs to meet the criteria of necessity, as though an aesthetic response is pre-set by a universal law of formal rightness.

This is a complex bureaucracy of conditions. Maybe it's their complexity that gives them their sticking power, or maybe it's the confidence by which they ask to be asserted, with the backing of a notion of universal truth. Either way they can still be found in the attentional expectations circulating around the reception of painting in the mid twentieth century and still today.

Rosalind Krauss has called these aesthetic conditions 'doubly paradoxical', the multiple and opposing demands they map, layer upon layer of unresolvable expectations; both subjective and 
universal, removed from conceptual concerns but necessarily communicable. To quote Krauss, aesthetic experience is 'conceived as pleasure disincarnated because without desire, and as pure individuality that can only act by assuming the assent of others' (1993: 114). Pierre Bourdieu, Terry Eagleton and more recently Jacques Ranciere, have commented on the ideological frameworks that construct aesthetic priorities, how the promotion of aesthetic sensibilities serve social and political interests (Bourdieu 1979; Eagleton 1990; Ranciere 2013) and we can acknowledge how disengagement and disinterest have social and political consequences. Eagleton also describes the double-handed conditions imposed, 'on the one hand a liberatory concern with concrete particularity, and on the other hand a specious form of universalism' (1990: 9).

In The Optical Unconscious, a parallel text to Painting as Model in terms of the task of extracting or salvaging a position from a certain strain of formalism, Rosalind Krauss describes a peculiar consequence of this matrix of conditions, recounting two versions of a modernist attentional sensibility, both held at different moments by Michael Fried. First, the super speed of a baseball player's vision, seeing faster than anyone else, being proposed as the ideal viewer of modernist painting (Krauss 1993: 6-8). And then the more often quoted 'presentness is grace' which values a suspended now, endlessly renewable, a decidedly theological precedent for an attentional attitude painting should value.

Both versions are based on the criteria of immediacy, the all at onceness of aesthetic experience, though articulated differently. Both rely on an understanding of the conditions of vision as abstract, and the influence of a notion of vision that is an objective capture of visual information. Neurologists in the nineteenth century were working from an assumption of a visual image formed on the retina that was passively and in its entirety, received by the cortex. This is no longer how visual processes are understood to work but the assumption that vision happens all at once, like 
the workings of a camera, were absorbed into a cultural understanding of vision, in the midst of burgeoning imaging technologies in the nineteenth century and onwards (Zeki 1993).

The esteem for the efficiency of a baseball player's response to the raw data of vision is one consequence of this. The other opens up a philosophical proposition that there is a natural or pure vision that we can aspire to, an image of the world that is complete and unfiltered, that leads to Fried's alternate articulation of immediacy that is vision in every instance made new.

As Krauss notes 'from the secularity of the baseball player to the metaphysics of grace is a leap indeed' and one that for Krauss produced 'a shudder like a lining ripping open so that the ideological seams showed through' (Krauss 1993: 8). Both versions value a mode of attention that propels painting into a narrowing corner of possibilities. One in a machine like insistence on essential information, one in evocation of something other than the deadpan fact of painting as a thing made.

Under these circumstances attention's role in perceptual synthesis gets condensed into demands for a philosophical wholeness and all at oneness.

In Whose Formalism? Yve Alain Bois has pointed out Clement Greenberg's 'indifference to the actual stuff of which any work of art is made' (1996), this indifference a sort of sublimation of matter, or in this context an example of inattentional blindness, an inability to perceive what is in plain sight. The attentional selectivity of Greenberg's formalism, with its notions of pure perception or isolated opticality rush over painting's materiality, its awkwardness as an object, its mode of thought and the speculative, shifting attention it might prompt. 
The attentional sensibilities evident in Michael Fried, and Clement Greenberg, and before them Clive Bell and Roger Fry are evidence of a desire to sustain what T. J. Clark has called 'a form of pristine experience had by an individual in front of an object' (1983). The isolation and singularity of this desire discounts a moving viewer, only allowing for one imagined stationary and transfixed, and it leads to a dismissal of modes of attention for painting that are more spatially dependent and discursive. Positioned in opposition to the directed attention of seriality, the attentional capture and distraction of comparative looking, or the dispersal of attention within an architectural volume, an unsustainably ideal model of attention can be implicated in the unravelling of modernist painting. This ideal model of attention detaches painting from the complexity, variability, scatter and stretch of attentional possibilities. I do not wish to discount the attentional experiences of any individual in front any object, but I do want to discount a sense of rightness or appropriateness in the attentional conditions of that encounter.

Bence Nanay's recent writing on aesthetic attention aims to reposition the qualities of aesthetic sensibility within the context of current cognitive research. Nanay proposes that the priorities of distributed attention can be seen in exemplars of aesthetic experience (2016). Distributed attention that we have seen contributes to our ability to estimate rather than count; that facilitate a perceptual all overness that we recognize as important to painting at particular points in its history. Nanay describes the predominant form of aesthetic attention in terms of distributed attention, and qualifies it as 'attention that is focused on one perceptual object, but it is distributed among the various properties of this object' (2015: 96). This compound of selectivity and dispersal is one that Nanay values, that there is pleasure in how the imprint of materiality and intention stretches our attentional resources in a particular way. 
When considering the question, what is the mode of attention of which painting is the stake?, I question an ideal model of attention that has been brought to bear on painting. The variability of attention is ultimately incompatible with a tightly bound, finely tuned attentional expectation. This incompatibility is, I argue, a factor in the unravelling of modernism that Bois's Painting as Model more widely tracks. Rather, as a structure invested in capturing and conditioning attention, painting requires the full spectrum of attentional modes. Attention does not settle in an attempted division between the optical and the cognitive. The processes of attention are culturally dependent and can be ideologically conditioned, and it is not useful to consider an attentional engagement with painting in isolation from other attentional interactions. Attention is both stimuli responsive and task responsive, as it negotiates the complexity of painting's prompts to visual cognition, and the speculative modalities of attention shift and adapt and shift again.

An acknowledgement of attention's variability is there in the art historical literature; Jonathan Crary writing on the gaps and discontinuities in a Paul Cezanne landscape, or Michael Baxandall accounting for the distracting agency of a nail on the upper periphery of a Georges Braque still life (Crary 1999) (Baxandall 1994). And attention's receptiveness to social and political considerations is present is Alois Riegl's particular use of the term 'attentiveness' which signifies a collective and socially responsible sense of perceptual engagement (Olin 1989; Riegl [1902] 2000). And the stretch and temporality of attentional possibilities is there in contemporary painter R. $\mathrm{H}$. Quaytman's reflection on paintings as objects you pass by, as things that you see 'not just head-on and isolated, but from the side, with your peripheral vision, and in the context of other paintings' (Quaytman 2010). And turning to Frank Stella at the last, there in the obstinate materiality and visual abrasiveness of the Black Paintings, like an attentional sticking point in any call for pure opticality. 
In an interview in 2009 with Michael Auping, Stella describes the material engagement of his making in the late 1950s and early 1960s. How the viscosity and drag of household paint on canvas slowed the process down, that 'you can't paint a straight line over that big of a surface' and the wobbliness of the brush mark line that allowed illusion in, however deadpan the delivery (Auping and Stella 2015). 50 years of remembering the particularity of a process, 50 years enacting equivalent processes which speculate on painting's possibilities, I will quote Frank Stella directly:

You need visual texture and tension to sustain the eye's attention. The thick lines and the patterns of the Black Paintings had that [...]. The eye likes dissonance. It's what keeps us looking. It makes us want to re-organize what we see [...]. (Auping and Stella 2015)

\section{References}

Auping, M. and Stella, F. (2015), 'The un-secret world of Frank Stella', VoCA Journal, Nov 122015 http://journal.voca.network/the-un-secret-world-of-frank-stella/ Accessed 7 February 2019.

Baxandall, M. (1994), 'Fixation and distraction: The nail in Braque's Violin and pitcher (1910)', in J. Onians (ed.), Sight and Insight: Essays on Art and Culture in Honour of E.H. Gombrich at 85, Phaidon Press, London, pp. 399-415.

Bois, Y. (1990), Painting as Model, MIT Press, Cambridge Massachusetts. . (1996), 'Whose formalism?' March 1996

https://www.mutualart.com/Article/Whose-formalism-/6B323BBF31A195F8 Accessed 7 February 2019.

Bourdieu, P. (1979), Distinction: A Social Critique of the Judgment of Taste, Harvard University Press, Cambridge Massachusetts.

Eagleton, T. (1990), The Ideology of the Aesthetic, Blackwell Publishers, Oxford. 
Chong, S. C. and Evans, K. K. (2011), 'Distributed vs. Focused Attention (count vs. estimate), WIREs Cognitive Science, November 2011, vol 2, issue 6, pp. 634-38,

https://www.ncbi.nlm.nih.gov/pmc/articles/PMC3222565/. Accessed 7 February 2019.

Clark, T. J. ([1983] 1985), 'Arguments about modernism: A reply to Michael Fried', in F. Frascina (ed.), Pollock and After: The Critical Debate, Harper \& Row, New York, pp.105-06.

Crary, J. (1999), Suspensions of Perception: Attention, Spectacle and Modern Culture, MIT Press, Cambridge Massachusetts.

Duttlinger, C. (2007), 'Between contemplation and distraction: Configurations of attention in Walter Benjamin', German Studies Review, 30:1, pp. 33-54.

Franconeri, S. L., Simons, D. J. and Junge, J. A. (2004), 'Searching for stimulus-driven shifts of attention', Psychonomic Bulletin \& Review, vol 11, issue 5, pp. 876-881.

Hamlin, A. J. (1896), 'Attention and distraction', The American Journal of Psychology, 8:1, pp. 3-66 (quotes from Marillier, M. L. [1889]).

Hayles, K. N. (2007), 'Hyper and deep attention: The generational divide in cognitive modes', Profession, Profession 2007, pp. 187-199.

James, W. (1890), The Principles of Psychology, 2 vols., chapter XL, Attention, New York: Holt, https://psychclassics.yorku.ca/James/Principles/prin11.htm.

Joselit, D. (2016), 'Marking, scoring, storing, and speculating (on time)', in I. Graw and E. Lajer-Burcharth (eds), Painting Beyond Itself: The Medium in the Post Medium Condition, Sternberg Press, Berlin

Krauss, R. (1993), The Optical Unconscious, The MIT Press, Cambridge Massachusetts. Nanay, B. (2015), 'Aesthetic attention', Journal of Consciousness Studies, 22:5\&6, pp. 96-118. (2016), Aesthetics as Philosophy of Perception, Oxford University Press, Oxford.

Olin, M. (1989), Forms of Representation in Alois Riegl's Theory of Art, The Pennsylvania State University Press, Pennsylvania. 
Prinz, J. J. (2012), The Conscious Brain: How Attention Engenders Experience, Oxford University Press, Oxford.

Quaytman, R. H. (2010), In the Studio: R.H. Quaytman, interviewed by Steel Stillman, Art in America, New York, 4 June,

https://www.artinamericamagazine.com/news-features/magazines/rh-quaytman/.

Ranciere, J. (2013), The Politics of Aesthetics, Bloomsbury Academic, London.

Riegl, A. ([1902] 2000), The Group Portraiture of Holland, Oxford University Press, Oxford.

Stone, L. (1998), 'Continuous partial attention',

https://lindastone.net/qa/continuous-partial-attention/ Accessed 07 February 2019

Terranova, T. (2012), 'Attention, economy and the brain', Culture Machine, vol 13, pp. 1-19.

Treisman, A. (2006), 'How the deployment of attention determines what we see', Visual Cognition, 14:4\&8, pp. 411-43.

Wyble, Folk, Potter (2012), 'Contingent attentional capture by conceptually relevant images', Journal of Experimental Psychology, Human Perception and Performance, 39:3, pp. 861-71.

Yantis, S. and Jonides, J. (1984), 'Abrupt visual onsets and selective attention: Evidence from visual search', Journal of Experimental Psychology, Human Perception and Performance, 10:5, pp. 601-21.

Zeki, S. (1993), A Vision of the Brain, Blackwell Scientific Publications, Oxford.

\section{Contributor details}

Moyra Derby is an artist and Senior Lecturer in Painting at the University for the Creative Arts.

Contact: mderby@uca.ac.uk

School of Fine Art and Photography, University for the Creative Arts, New Dover Road, Canterbury, CT1 3AN 